\title{
The Danish National Prescription Registry
}

Kildemoes, Helle Wallach; Toft Sørensen, Henrik ; Hallas, Jesper

Published in:

Scandinavian Journal of Public Health

DOI:

$10.1177 / 1403494810394717$

Publication date:

2011

Document version

Early version, also known as pre-print

Citation for published version (APA):

Kildemoes, H. W., Toft Sørensen, H., \& Hallas, J. (2011). The Danish National Prescription Registry.

Scandinavian Journal of Public Health, 39(suppl 7), 38-41. https://doi.org/10.1177/1403494810394717 


\title{
The Danish National Prescription Registry
}

\author{
HELLE WALLACH KILDEMOES ${ }^{1,2}$, HENRIK TOFT SØRENSEN ${ }^{3}$ \& JESPER HALLAS ${ }^{4}$ \\ ${ }^{1}$ Center for Healthy Ageing, Department of Public Health, University of Copenhagen, Denmark, ${ }^{2}$ National Institute of Public \\ Health, University of Southern Denmark, Denmark, ${ }^{3}$ Department of Clinical Epidemiology, Aarhus University Hospital, \\ Denmark, and ${ }^{4}$ Department of Clinical Pharmacology, Institute of Public Health, University of Southern Denmark
}

\begin{abstract}
Introduction: Individual-level data on all prescription drugs sold in Danish community pharmacies has since 1994 been recorded in the Register of Medicinal Products Statistics of the Danish Medicines Agency. Content: The register subset, termed the Danish National Prescription Registry (DNPR), contains information on dispensed prescriptions, including variables at the level of the drug user, the prescriber, and the pharmacy. Validity and coverage: Reimbursement-driven record keeping, with automated bar-code-based data entry provides data of high quality, including detailed information on the dispensed drug. Conclusion: The possibility of linkage with many other nationwide individual-level data sources renders the DNPR a very powerful pharmacoepidemiological tool.
\end{abstract}

Key Words: Denmark, dispensed prescription drugs, individual-level data, nation-wide register

\section{Introduction}

Since 1994, information on all prescription drugs sold in Denmark has been recorded in the Register of Medicinal Products Statistics (RMPS), maintained by the Danish Medicines Agency [1]. From an international perspective, the RMPS is unique, providing individual-level information on dispensed prescriptions for an entire nation since 1994. Similar nationwide prescription registers have afterwards been established in other Nordic countries [2].

In addition to prescription information, RMPS contains aggregate data on sales of over-the-counter (OTC) drugs and drugs sold for inpatient use. The RMPS was established by law in order to create drug price indexes and compile statistics for health authorities. Subsequent legalization enabled the use of the individual-level data for research. Since 2003, the prescription data in RMPS have been made available for researchers through an encrypted copy of the entire prescription register - except for the 1994 data, which are incomplete. This sub-register, administered by Statistics Denmark, is termed the Danish National Prescription Registry (DNPR). The tracking of individual prescription histories over time is made feasible by a unique personal identification number (CPR-number), assigned to all residents at birth or upon immigration, and it is included in all national registers $[3,4]$.

As described elsewhere in this supplementum, researchers at authorized institutions can apply for online access to extracts of individual-level registers maintained or administered by Statistics Denmark, including DNPR [5]. Before releasing data for research, Statistics Denmark encrypts the original CPR-number, permanently replacing it with another identifier.

\section{Content}

DNPR contains information on all prescription drugs dispensed at Danish community pharmacies. Unlike prescription registers in other countries, the

Correspondence: Helle Wallach Kildemoes, Section for Health Services Research, Department of Public Health, University of Copenhagen, CSS, Øster Farimagsgade 5, DK-1014 Copenhagen, Denmark. E-mail: hewk@sund.ku.dk 
DNPR contains individual-level information on prescriptions dispensed to residents of long-term care institutions, such as nursing homes.

The variables in DNPR can be divided into four main categories: variables related to drug user, to prescriber, and drug and pharmacy variables.

(1) The individual drug user is identified by the encrypted CPR-number. The person identifier allows tracking of all prescriptions redeemed by the same individual and enables linkage of prescription data to other individual-level registers managed by Statistics Denmark, such as socio-demographic and inpatient registers.

(2) Information on prescribers is organized by their practice or hospital department, and linked to information on medical specialty (e.g., general practitioner, hospital-based gastroenterologist). From 2003, the prescriber practice code has been made available for researchers, provided no linkage is made to identify the physician's community.

(3) Each dispensed drug is identified by the Nordic article number [6] of the drug formulation, which codes information on trade name, pharmaceutical form, strength, and package size. Through a linkage to the World Health Organization's Collaborating Centre for Drug Statistics Methodology [7], it is possible to establish the global Anatomical Therapeutic Chemical classification (ATC) code, identifying the dispensed drug in a five-level classification system, and the defined daily dose (DDD). The DDD of the drug is its assumed average adult daily maintenance dose administered for the main indication as mono-therapy. The amount of drug dispensed is expressed in terms of number of DDDs per package, enabling researchers to assess trends in drug consumption for different drugs within the same category. More than 30 additional categories of drug information are available in the DNPR, including dispensing date, number of packages dispensed, number of units per packet, dosage form and strength, availability (e.g. dose dispensing), pharmacy retail price, patient co-payment, and reimbursement codes.

(4) The individual pharmacies are identified by a code.

A selection of the variables included in DNPR is listed in Table I. The non-included variables are mainly related to the administrative handling of the prescription or its re-imbursement. They are available from Statistics Denmark upon request.

Although important for some researchers, DNPR does not include the following information:

(i) Drugs sold without a prescription are not recorded on an individual level. Thus, OTC purchasing is generally not registered in DNPR. Yet,
Table I. Selected variables available in the Danish National Prescription Register.

\begin{tabular}{ll}
\hline Variables related to & \multicolumn{1}{c}{ Contents } \\
\hline Drug user & Personal identification number (CPR-number) \\
& Age at dispensing date \\
& Gender \\
& Municipality of residence \\
& Region of residence \\
Dispensing & Date of dispensing \\
& Product code (Nordic article number) \\
& Product name \\
& Anatomical Therapeutic Chemical \\
& classification (ATC) code \\
& Dose unit \\
& Number of dose units in package \\
& Retail price \\
& Number of prescription refills \\
& Reimbursed amount and type \\
& Dosing instruction (optional, in free text) \\
& Indication for prescribing (optional, \\
& in free text) \\
Prescriber & Prescriber practice code \\
Pharmacy & Dispensing pharmacy code \\
\hline
\end{tabular}

Non-included variables mainly relate to the administrative handling of the prescription.

OTC drugs may be dispensed on prescription for chronic diseases to entitle the patient for reimbursement, in which case they are recorded on the individual level.

(ii) Drugs dispensed at hospitals for outpatient treatment, such as anti-neoplastic drugs or HIV drugs, are not registered in DNPR.

(iii) DNPR contains information only on redeemed prescriptions. Thus, it does not document prescriptions issued by a physician but not filled ("primary non-compliance").

(iv) The prescribed daily dose and the indication for prescribing the drug have recently been added as variables. Yet, the non-mandatory information is entered as free text, which is not readily usable for research.

DNPR has undergone only minor changes since its initiation. All Danish pharmacies are still obliged to register dispensed prescriptions electronically for RMPS. Before 1996, drugs prescribed for children under age 16 were registered as dispensed for their mother (i.e. under her CPR-number). Since 1 January 1996 drugs prescribed for children have been registered under the child's own CPR-number.

\section{Coverage and validity}

The DNPR contains data on all prescriptions dispensed for Danish residents at outpatient 
pharmacies. The linkage through the unique CPR-number to other individual-level data sources enables researchers to identify and follow prescriptions on the individual level over time at all Danish pharmacies. Thus, for individuals with permanent residence in Denmark, loss to follow-up is unlikely.

Due to the closed-circuit character of the register, few studies have assessed the completeness and validity/reliability of DNPR by means of an external standard $[8,9]$. Yet, several factors point towards a high data quality.

As opposed to the physician's recording of inpatient data (diagnoses etc.) in administrative systems, the registering of data for DNPR constitutes an integral component of a pharmacist's key function of selling prescription drugs. This includes maintaining the computerized reimbursement account on the individual level. For all Danish inhabitants, the actual amount of reimbursement depends on whether the particular drug is reimbursable (decided by the Danish Medicines Agency at the National Board of Health) and on preceding out-of pocket expenses on reimbursable drugs [10]. Thus, the universal reimbursement system provides a strong economic incentive for recording all drugs dispensed. Increasing use of internet pharmacies to obtain low-price drugs may, however, undermine the completeness of DNPR if a substantial proportion of dispensed prescriptions begins to bypass registration.

All drug-packets are labelled with an optically scanned barcode corresponding to the Nordic article number that acts as linkage to other registers containing all necessary information about the drug. Thus, inter/intra observer and information bias are very unlikely to occur in DNPR. In contrast to assessment of medical diagnoses that may vary substantially across medical practitioners, within the same medical practitioner and over place/time (due to time-dependent diagnostic criteria/procedures and administrative practices), sold drugs are uniquely defined through the barcode.

Generally, the concordance of register-based and questionnaire-based data on drug use have been reassuring $[8,9]$. Yet, the validity depends on the extent to which the prescription information captures what it was intended to measure in the particular pharmacoepidemiological study. Thus, for drug exposure studies the concern lies in the reliance on prescription redemption as a surrogate measure for the actual ingestion of the drug by the person for whom it was prescribed. However, when redeemed regularly, the drug is most likely being ingested. When studying prescribing behaviour, the uncertainty lies in the fact that prescriptions are not necessarily redeemed ("primary non-compliance").

\section{Pharmacoepidemiological research and regional prescription registers}

The development of the pharmacoepidemiological discipline in Denmark was pioneered at two research institutions: the Department of Clinical Epidemiology at Aarhus University and the Department of Clinical Pharmacology/General Practice at the University of Southern Denmark, Odense [11]. In Aarhus, the research has primarily focused on aetiological studies of adverse drug reactions, with particular emphasis on fatal drug exposure, whereas in Odense the focus has been on drug utilization, quality indicators, and prescriber feedback. Each institution has developed its own regional prescription register, covering the County of Northern Jutland from 1989 and the County of Funen from 1990, respectively. Both registers are active today and have extended their coverage to neighbouring regions.

The regional registers and the DNPR differ in several important ways. While the DNPR includes all prescriptions regardless of reimbursement status, the regional registers exclusively cover reimbursable prescription drugs. Consequently, non-reimbursable prescription drugs, such as hypnotics and benzodiazepines, oral contraceptives, and certain antibiotics, are not recorded in the regional registers. On the other hand, the regional registers are considerably more "open" than the DNPR. These registers are allowed to give person-identifiable data to researchers upon approval from the Danish Data Protection Agency. This opens possibilities for a wider range of epidemiological studies on identifiable drug users and permits validation of recorded prescription data using external sources. Furthermore, it is possible to use the regional data to provide feedback to prescribers. The DNPR's closed-circuit safety approach precludes these activities. Finally, both regional registers were established earlier the DNPR.

\section{Conclusion}

The Danish National Prescription Registry is one of the few individual-level prescription registers that cover an entire nation [2]. With its high quality prescription data since 1995, and the potential for linkage with other data sources, the register is potentially one of the world's most powerful pharmacoepidemiological tools. 


\section{Funding}

This research was supported by a grant from the Nordea Foundation for the Center for healthy Ageing.

\section{References}

[1] The Danish Medicines Agency. Available at: http:// www.dkma.dk (accessed March 2010).

[2] Furu K, Wettermark B, Andersen M, Martikainen JE, Almarsdottir AB, Sorensen HT. The Nordic countries as a cohort for pharmacoepidemiological research. Basic Clin Pharmacol Toxicol 2010;106(2):86-94.

[3] Frank L. Epidemiology. When an entire country is a cohort. Science 2000;287(5462):2398-9.

[4] Petersen CB. The Danish Civil Registration System. Scand J Public Health 2011;39(Supp1 7):22-5.

[5] Thygesen LC, Daasnes C, Thaulow I, Brønnum-Hansen H. Introduction to Danish (nationwide) registers on health and social issues: Structure, access, legislation, and archiving. Scand J Public Health 2011;39(Suppl 7):12-6.

[6] The Nordic Article Number. Available at: http://www. laaketeollisuus.fi/tiedostot/Vnr_Ohjekirja_Eng_2007.pdf (accessed March 2010).

[7] WHO Collaborating Centre for Drug Statistics Methodology. Norwegian Institute of Public Health; Available at: http://www.whocc.no/atc_ddd_index (accessed March 2010).

[8] Wogelius P, Poulsen S, Sorensen HT. Validity of parentalreported questionnaire data on Danish children's use of asthma-drugs: a comparison with a population-based prescription database. Eur J Epidemiol 2005;20(1):17-22.

[9] Lokkegaard EL, Johnsen SP, Heitmann BL, Stahlberg C, Pedersen AT, Obel EB, et al. The validity of self-reported use of hormone replacement therapy among Danish nurses. Acta Obstet Gynecol Scand 2004;83(5):476-81.

[10] Pedersen KM. Pricing and reimbursement of drugs in Denmark. Eur J Health Econom 2003;4:60-5.

[11] Hallas J. Conducting pharmacoepidemiologic research in Denmark. Pharmacoepidemiol Drug Saf 2001;10(7): 619-23. 